\section{(6) OPEN ACCESS}

\title{
Work-focused cognitive-behavioural therapy and individual job support to increase work participation in common mental disorders: a randomised controlled multicentre trial
}

\author{
Silje Endresen Reme, ${ }^{1}$ Astrid Louise Grasdal, ${ }^{2}$ Camilla Løvvik, ${ }^{1,4}$ Stein Atle Lie, \\ Simon Øverland $d^{3,4}$
}

- Additional material is

published online only. To view please visit the journal online (http://dx.doi.org/10.1136/ oemed-2014-102700).

${ }^{1}$ Uni Research Health, Bergen, Norway

${ }^{2}$ Department of Economics, University of Bergen, Bergen, Norway

${ }^{3}$ Department of Public Mental Health, Norwegian Institute of Public Health, Bergen, Norway ${ }^{4}$ Department of Psychosocial Science, University of Bergen, Bergen, Norway

Correspondence to Dr Silje Endresen Reme, Uni Health, Uni Research, Krinkelkroken 1, Bergen 5020, Norway; silje.reme@uni.no

Received 12 November 2014 Revised 24 April 2015 Accepted 22 June 2015 Published Online First 10 August 2015

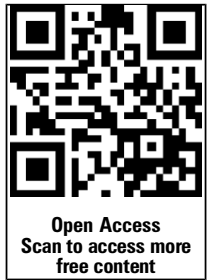

\section{ABSTRACT}

Objectives Common mental disorders (CMDs) are a major cause of rising disability benefit expenditures. We urgently need evidence on programmes that can increase work participation in CMDs. The aim of this study was to evaluate the effectiveness of work-focused cognitivebehavioural therapy (CBT) and individual job support for people struggling with work participation due to CMDs. Methods A randomised controlled multicentre trial (RCT) including 1193 participants was conducted. Participants were on sick leave, at risk of going on sick leave or on long-term benefits. The intervention integrated work-focused CBT with individual job support. The control group received usual care. The main outcome was objectively ascertained work participation at 12 months follow-up, with changes in mental health and health-related quality of life as secondary outcomes. Results A larger proportion of participants in the intervention group had increased or maintained their work participation at follow-up compared to the control group $(44.2 \%$ vs $37.2 \%, p=0.015)$. The difference remained significant after 18 months (difference $7.8 \%$, $\mathrm{p}=0.018$ ), and was even stronger for those on long-term benefits (difference $12.2 \%, p=0.007$ ). The intervention also reduced depression $(t=3.23, p \leq 0.001)$ and anxiety symptoms ( $t=2.52, p=0.012)$ and increased healthrelated quality of life ( $t=2.24, p=0.026)$ more than usual care.

Conclusions A work-focused CBT and individual job support was more effective than usual care in increasing or maintaining work participation for people with CMDs. The effects were profound for people on long-term benefits. This is the first large-scale RCT to demonstrate an effect of a behavioural intervention on work participation for the large group of workers with CMDs. Trial registration number ClinicalTrials.gov, registration number: NCT01146730.

\section{INTRODUCTION}

Sickness absence with mental disorders as a primary diagnosis has increased markedly over the past decade, and mental and behavioural disorders now account for more incapacity benefit claims than any other disorders, ${ }^{1} 2$ despite no clear increase in corresponding prevalence rates. ${ }^{3-5}$ Common mental disorders affect one-sixth of the working age population at any one point, ${ }^{6}$ many of

\section{What this paper adds}

- Common mental disorders are a major cause of rising disability benefit expenditures.

- We urgently need evidence on programmes that can increase work participation in common mental disorders.

- An integrated model of work-focused cognitive-behavioural therapy and individual job support, emphasising early re-entry, was more effective than usual care in increasing or maintaining work participation for people with common mental disorders.

- The effects were profound for people on long-term benefits.

whom struggle to cope with and retain their place in working life. ${ }^{17}$ As a consequence, these disorders represent a significant loss of labour supply and increase state expenditure through sickness absence and disability pensions. ${ }^{1}$ With longer sickness absences, it becomes less likely that the person will return to work (RTW). ${ }^{8}{ }^{9} \mathrm{~A}$ crucial challenge is therefore to disrupt the process of prolonged sickness absence before it progresses into permanent disability. ${ }^{7}$

Cognitive-behavioural therapy (CBT) is established as standard treatment for depression ${ }^{10}$ and anxiety. ${ }^{11}$ There is, however, no one-to-one relationship between disorder, symptom levels and work participation, ${ }^{7}$ and it is not clear if treatment and remission of a common mental disorder, such as by way of CBT, results in increased work participation for the individual. Occupational rehabilitation has often followed a 'two-phased approach', with an initial medical goal of alleviating health problems, whereupon the individual with or without further assistance is expected to RTW. Recent evidence challenges this as early re-entry to ordinary employment with close follow-up can increase both work participation ${ }^{12-15}$ and mental health outcomes ${ }^{16}$ in severe mental disorders. It is not known if this approach can be extended to common mental disorders. In the light of the high prevalence of common mental disorders and their 
major impact on sickness absence and disability pension award, efficacious interventions to restore working capacity and prevent ill health retirement for this patient group are needed.

The main objective of this study was to evaluate the effectiveness of an integrated model (work-focused CBT and individual job support), labelled AWaC (At Work and Coping), for people struggling with work participation due to common mental disorders. The effect was evaluated in a pragmatic randomised controlled multicentre trial (RCT), to reach robust conclusions in an ecologically valid study setting. Participants were randomised to AWaC or usual care provided by the Norwegian Labor and Welfare Administration (NAV) and/or general practitioners (GPs).

Primary objective: (1) To investigate the main effect of AWaC on work participation at 12 months follow-up.

Secondary objectives: (1) to investigate changes in mental health and health-related quality of life at 12 months follow-up; (2) to calculate the cost-effectiveness of $\mathrm{AWaC}$ and (3) to examine effect differences in subgroups defined by gender, age, baseline work status, health variables and time of inclusion.

\section{METHODS}

\section{Study context}

The Norwegian public insurance system includes all lawful residents of Norway and provides health service benefits and pensions for all members of the National Insurance Scheme, administered by the Norwegian Welfare and Labor Administration (NAV). The workers' compensation programme is part of the scheme and provides $100 \%$ coverage for lost income due to medically acknowledged sickness, disease or injury (including mental illness) from day one until the person can work again, up to 52 weeks. After that, long-term benefits provide approximately $66 \%$ of former income.

\section{Procedure}

People aged 18-60 years who were struggling with work participation attributable to common mental disorders were invited. This included people on and at risk of sick leave, as well as people on long-term benefits (primarily participants on work assessment allowance after $>12$ months sick leave). During the first assessment of eligibility, a clinical psychologist assessed the presence of common mental disorders. This assessment was based on informal self-reports of symptoms and symptoms consistent with anxiety and/or depression. This included the subthreshold symptoms of anxiety and depression disorders, as they too are strongly associated with functional disability and absence from work. ${ }^{17}$ Eligible participants had to express a motivation to RTW/stay at work. Participants were excluded if reasons other than common mental disorders were the primary cause of problems with work participation, such as no motivation to participate in working life; severe psychiatric disorders; high suicide risk; pregnancy and ongoing substance abuse. Inability to read Norwegian and engagement in psychotherapy elsewhere also led to exclusion.

1416 potential participants were referred and considered for inclusion (6/1/2010-2/1/2012). Of these, 197 did not fulfil the inclusion criteria, 17 did not consent to participate, and 9 withdrew their consent and required data deletion (see online supplementary figure). In total, 1193 participants entered the trial and were randomised, 52.8\% $(\mathrm{n}=630)$ to the intervention group and $47.2 \%(n=563)$ to the control group. The unbalanced allocation (a 2:1 randomisation ratio in the first 5 months of recruitment) ensured that the centres could run according to maximal capacity. The randomisation procedure strictly adhered to the formal requirements of adequate randomisation at all times (see online supplementary file for the full trial protocol).

Of the 1193 participants, 336 (32\%) were referred from NAV, $238(23 \%)$ from their GP, 351 (22\%) were self-referred, 124 $(12 \%)$ got referred from other service providers, and 144 participants did not inform on the pathway to the trial.

Potential participants were informed about the project and screened for inclusion and exclusion criteria at the centres by a clinical psychologist. Eligible and willing participants signed the informed consent and completed the baseline questionnaire. After random allocation, participants were informed about the outcome, and the intervention group was given a date for its first session.

\section{Ethical considerations}

The research was carried out in compliance with all principles in the Helsinki declaration. Personal confidentiality was guaranteed, and informed consent was signed by each participant with emphasis on the right to withdraw from the study at any time without any explanation.

\section{Interventions}

The AWaC programme provides a systematic approach where individual CBT and job support are integrated. Miniteams of therapists and employment specialists were formed at each centre to ensure integration between CBT and the explicit work focus. CBT was characterised by 'cognitive work-coping', and focused on managing mental health problems as they relate to work situations. Up to 15 sessions of CBT were offered. The individual job support was based on the 'Individual Placement and Support (IPS)' approach, developed for people with severe mental illness, ${ }^{18}$ and was offered to those in need of individual job support (primarly participants on long-term disability) to facilitate workplace adaptations or identification of appropriate employment. IPS represents a relatively new approach to vocational rehabilitation and incorporates the following eight principles: eligibility based on consumer choice, focus on competitive employment, integration of mental health and employment services, attention to client preferences, work incentives planning, rapid job search, systematic job development and individualised job support. The IPS framework is less specific on choice of therapeutic approach within the mental health services. The integration of the IPS principles and work-focused CBT in the AWaC thus represents a unique and innovative elaboration of the IPS model for people with common mental disorders (extensive information available in the study protocol, online supplementary file, pp.23-37).

Patients allocated to the control group received standard treatment from their GP, national insurance office (NAV), other health professionals, and received a letter with information and encouragement to use available services and self-help resources. Employment and health care services for the control group were not restricted (beyond ruling out the $\mathrm{AWaC}$ ), they could well be followed up by other psychologists and/or participate in other employment schemes initiated by NAV. The control condition thus represents an active, as opposed to passive, control condition, and illustrates how the main distinguishing feature of the $\mathrm{AWaC}$ is the systematic and integrated approach.

\section{Outcomes}

Using data from the national social insurance register and the national employee register, we could (with no loss to follow-up) determine if a person (1) was in regular work (part time or full 
time), (2) was in combined work and a recipient of benefits, or (3) was out of work, with or without social insurance benefits. The primary outcome was 'increased or maintained work participation' at 12 months post baseline, operationalised as maintained work participation, new employment or a full or partial RTW, depending on the individual's baseline work status. Full or partial RTW was operationalised as: working and no reception of health-related or work-related benefits, or reduced benefit coverage and increased work participation compared with baseline status. This information was collected for each month of follow-up, up until 18 months, to examine sensitivity of the primary 12 months results.

The secondary outcome measures were questionnaire-based changes in psychological distress, and symptoms of anxiety and depression by use of the Hospital Anxiety and Depression (HAD) Scale. ${ }^{19} 20$ EQ5D was used to measure changes in health-related quality of life. ${ }^{21}$ Self-report questionnaires were administered to the patients at baseline and after 6 and 12 months.

\section{Sample size}

The commisioning body predicted that the AWaC centres would recruit 600-1000 participants/year. On the basis of an optimistic $8 \%$ effect difference in favour of $\mathrm{AWaC}$ versus control $(48 \%$ vs $40 \%$ increased work participation), we calculated that a sample size of 600 in each group would be sufficient to detect statistically significant results.

\section{Randomisation}

The participants were randomised with a computer-generated randomisation list stratified by centre. At each centre, the person responsible for inclusion emailed the research unit, presented the participant's project ID number, name and gender before treatment allocation was disclosed. The allocation code, including details of block size (10), was not revealed to the researchers or the clinicians until recruitment and data collection were complete.

\section{Statistical methods}

For the main effect analysis, we observed crude rates of participants with increased work participation in the two groups. The effects of treatment were further examined by logistic and multinomial logistic regression analyses when adjusting for minor by-chance remaining differences in observed characteristics between the intervention group and the control group. 95\% CIs were based on SEs obtained from logistic and multinomial logistic regressions estimated separately for each calendar month during 12-18 months after inclusion and corrected for clustering on site. For the secondary outcomes (mental health), we performed analyses with inverse probability weights ${ }^{22}$ to account for possible attrition bias. Analyses adhered to the 'intention-to-treat' principle. The authors in charge of the data analysis were blinded for intervention assignment. Data were analysed using STATA V.12.

To test for potential subgroup effects of the $\mathrm{AWaC}$ programme, characteristics prespecified in the protocol (gender and age, work status at baseline, inclusion early vs late in the project period, and also duration and intensity of mental health symptoms) were entered in the regression model and assessed for potential influence on the main effect. Baseline work status was considered of particular importance and the effects are also presented in tables stratified on this variable.

\section{Treatment adherence}

Only $5 \%$ dropped out of treatment (completing $<3$ sessions) in the $\mathrm{AWaC}$ group. Treatment adherence in the control group was not registered.

\section{Eligibility criteria for care providers}

All therapists were monitored, videotaped and scored according to the Cognitive Therapy Adherence and Competence Scale ${ }^{23}$ prior to the trial enrolment. The AWaC CBT protocol was clearly defined from the outset. The employment specialists were required to have relevant qualifications and broad experience with supported employment, and extensive knowledge regarding the IPS principles ${ }^{24}$ and the job market in the team's region.

\section{Details on capacity}

Maximum client capacity for the six centres varied. At the most, 85 clients were enrolled at one centre, whereas the peak for the lowest ranking centre was 43 enrolled at the same time.

\section{Cost-benefit analysis of the impact of treatment}

We calculated the economic returns of $\mathrm{AWaC}$ compared with usual treatment by a standard cost-benefit formula based on the human capital approach. ${ }^{25-27}$ Economic benefits from treatment were calculated by multiplying treatment effects by the discounted net present value of productivity gains when a person works instead of receiving disability pension. Treatment effects were measured as the observed differences in RTW between the $\mathrm{AWaC}$ and the control group, averaged over the 12-18 months after enrolment. The estimate of the net present value of productivity gains were based on means of the group-specific observed annual income the year before enrolment, measured in Norwegian Krone (NoK) 2012. Estimates of productivity gains from work were based on annual income the year before inclusion. For a single worker, the productivity gain per year is the sum of annual gross earnings and social costs of labour, the latter represented by the payroll tax paid by the employer. In compliance with official recommendations (Ministry of Finance, 2005), the marginal cost of public funds was set at 0.2, whereas the social discount rate was set at 0.04 .

Costs of the $\mathrm{AWaC}$ programme included the total cost of treatment and follow-up at the centres, as well as the cost of additional treatment and follow-up by NAV and/or primary healthcare providers such as GPs, psychologists, physiotherapists and chiropractors. The costs of usual care included costs of treatment and follow-up by NAV and/or healthcare providers. Information about additional treatment and follow-up by NAV was collected from registers while information about healthcare services was collected through follow-up questionnaires. Information about costs per month for services and participation in labour market programmes provided by NAV, and about costs per consultation for healthcare services, were obtained from NAV.

Calculations of costs and benefits were based on annual production estimates when assuming that the centres can treat and follow-up 615 persons/year. Costs were measured in 2011 (index year). Benefits were calculated in 2012 and discounted to 2011. We performed an external economic evaluation based on the overall effect, as well as an internal economic evaluation based on the effect observed for participants who had been out of work for more than 12 months. While the former yields the net economic returns of the experiment, the latter approximates the economic returns of treatment if given only to persons on long-term benefits, who otherwise fulfilled the inclusion criteria used in the current study. 


\section{RESULTS}

\section{Loss to follow-up}

Data on the main outcome measure, work participation, were complete for all participants. However, for secondary outcomes based on self-report, $740(62 \%)$ participants at 6 months follow-up and 636 (52\%) participants at 12 months follow-up returned their questionnaires (see flow chart, online supplementary figure).

\section{Participant characteristics}

More women (67\%) than men (33\%) were referred and included in the trial. Mean age was 40.4 years (95\% CI 39.9 to 41.0) and on average participants reported having had psychological symptoms for 8.6 years $(\mathrm{SD}=9.76)$. At baseline, $31.4 \%$ of the participants were working (of these, $48 \%$ were combining work and sick leave), 39\% were fully on sick leave, $21.7 \%$ were on long-term benefits (>12 months sick leave) and $7.9 \%$ were unemployed (table 1). In the subgroup analyses, the unemployed people are included in the group on long-term benefits.

\section{Implementation of intervention}

In the AWaC group, all received CBT delivered by a clinical psychologist/counsellor, and 32\% also received individual job support. In addition, many also received other interventions from NAV and health services (see online supplementary table S1). Services provided for controls may have assimilated the intervention, but as the key element of AWaC was the integration of CBT and individual job support, the degree and impact of potential contamination was considered minimal.

Qualitative assessments in the early phase of the trial suggested challenges with implementation of the job support elements, but no fidelity assessments using the IPS fidelity scale ${ }^{24}$ were conducted at baseline as the employment intervention was being developed throughout the trial. Fidelity was, however, assessed postrecruitment, with results showing satisfactory quality and adherence to IPS. Still, there was no enrolment time $\times$ group allocation interaction $(\mathrm{p}=0.207)$.

In the control group, NAV deployed a total of 255 interventions in 157 individuals, the most common being work with assistance, labour market courses, traineeship and educational interventions. The AWaC protocol did not restrict use of other interventions, and the corresponding figures for the $\mathrm{AWaC}$ group were 206 interventions for 143 individuals. The control group reported a higher use of health services than the $\mathrm{AWaC}$ group (see online supplementary table S1).

\section{Number of care providers performing the treatment in each group}

The average number of employment specialists during the trial period was 5.7 full-time equivalents (FTEs) distributed across

Table 1 Baseline characteristics of participants

\begin{tabular}{|c|c|c|c|c|}
\hline & \multicolumn{2}{|l|}{ AWaC } & \multicolumn{2}{|l|}{ Control } \\
\hline & $\mathrm{n}$ & Per cent & $\mathrm{n}$ & Per cent \\
\hline Female & 437 & 69.37 & 365 & 65.01 \\
\hline Married & 189 & 30.00 & 184 & 32.68 \\
\hline \multicolumn{5}{|l|}{ Age (years) } \\
\hline$<30$ & 105 & 16.66 & 87 & 15.45 \\
\hline $30-39$ & 220 & 34.92 & 169 & 30.01 \\
\hline $40-49$ & 198 & 31.42 & 185 & 32.85 \\
\hline $50+$ & 107 & 16.98 & 122 & 21.66 \\
\hline \multicolumn{5}{|l|}{ Education } \\
\hline Primary & 49 & 7.78 & 36 & 6.39 \\
\hline Senior high & 196 & 31.11 & 187 & 33.21 \\
\hline University/college & 353 & 56.03 & 304 & 54.00 \\
\hline Other & 30 & 4.76 & 35 & 6.22 \\
\hline Missing data & 2 & 0.32 & 1 & 0.18 \\
\hline \multicolumn{5}{|l|}{ Self-assessed health } \\
\hline Good & 236 & 37.46 & 208 & 36.94 \\
\hline Medium & 301 & 47.78 & 248 & 44.05 \\
\hline Poor & 86 & 13.65 & 104 & 18.47 \\
\hline Missing data & 7 & 1.11 & 3 & 0.53 \\
\hline \multicolumn{5}{|l|}{ Employment status } \\
\hline \multicolumn{5}{|l|}{ Work } \\
\hline Work, no benefits & 104 & 16.51 & 88 & 15.63 \\
\hline Combined work and sick leave benefits & 95 & 15.10 & 88 & 15.63 \\
\hline Fully on sick leave & 254 & 40.16 & 210 & 37.30 \\
\hline Long-term health benefits & 131 & 20.79 & 128 & 22.74 \\
\hline \multirow[t]{2}{*}{ Unemployed } & 46 & 7.30 & 49 & 8.70 \\
\hline & Mean & $95 \% \mathrm{Cl}$ & Mean & $95 \% \mathrm{Cl}$ \\
\hline Anxiety (HADS) & 10.51 & 10.21 to 10.82 & 10.86 & 10.52 to 11.20 \\
\hline Depression (HADS) & 7.76 & 7.45 to 8.07 & 8.44 & 8.10 to 8.78 \\
\hline HADS total score & 18.27 & 17.74 to 18.80 & 19.29 & 18.71 to 19.88 \\
\hline
\end{tabular}

AWaC, At Work and Coping; HADS, Hospital Anxiety and Depression Scale. 


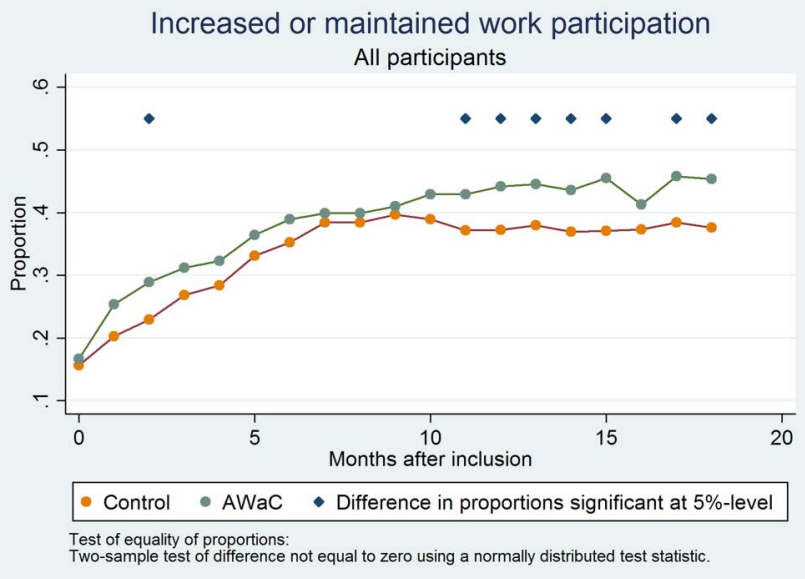

Figure 1 Full sample. Observed difference in proportions with increased or maintained work participation, intervention versus controls (AWaC, At Work and Coping).

nine people. The average number of therapists providing the work-focused CBT was 20 (20 FTEs), with each therapist treating 30 participants on average during the trial period.

\section{Primary outcome measure}

At 12 months, the proportion with increased or maintained work participation was higher in the AWaC group than in the control group $(44.2 \%$ vs $37.2 \%$, difference $6.9 \%, p=0.015)$, and this difference remained at 18 months (difference $7.8 \%$, $\mathrm{p}=0.018$; figure 1$)$. The effect difference remained statistically significant in adjusted models at 12 months (table 2).

The difference between the AWaC group and the control group was largest for those on long-term benefits at baseline, with $24 \%$ increased or maintained work participation in the AWaC group compared with $12 \%$ in the control group at 12 months (figure 2). The difference increased to $30 \%$ versus $11 \%$ at 18 months follow-up. There was no statistically

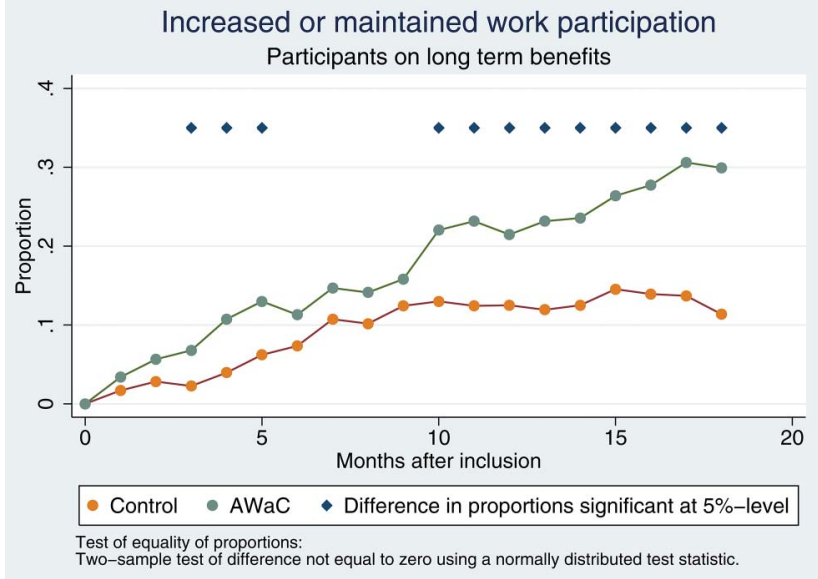

Figure 2 Participants on long-term benefits. Observed differences in proportions with increased or maintained work participation, intervention versus controls (AWaC, At Work and Coping).

significant effect difference between $\mathrm{AWaC}$ and controls in the other subgroups (on sick leave and at risk of going on sick leave). There were no significant effect differences in subgroup analyses based on gender, age, time of inclusion or health status at baseline.

\section{Secondary outcome measures}

The AWaC group reported significant improvements in mental health and a higher mean health-related quality of life at the 12-month follow-up (table 3), also after control for baseline scores. Adjusting for attrition using inverse probability weights based on demographics (age, gender, education) and the outcomes of interest (psychological distress, anxiety and depression symptoms) did not alter the results.

We observed no increased effect for the subgroup on longterm benefits regarding symptoms of mental health or health-related quality of life.

Table 2 Effects of treatment in AWaC versus treatment as usual on probability of increased or maintained work participation, calculated from logistic and multinomial regression estimates. Marginal effects, evaluated at sample mean of regressors

\begin{tabular}{|c|c|c|c|c|c|c|}
\hline \multirow[b]{2}{*}{ Month } & \multicolumn{2}{|c|}{ Increased/maintained work } & \multicolumn{2}{|l|}{ Fully in work } & \multicolumn{2}{|l|}{ Partly in work } \\
\hline & Marginal effect & $95 \% \mathrm{Cl}$ & Marginal effect & $95 \% \mathrm{Cl}$ & Marginal effect & $95 \% \mathrm{Cl}$ \\
\hline \multicolumn{7}{|c|}{ All participants } \\
\hline 12 & 0.062 & 0.005 to 0.118 & 0.034 & -0.026 to 0.095 & 0.025 & -0.014 to 0.064 \\
\hline 13 & 0.056 & 0.000 to 0.113 & 0.035 & -0.023 to 0.093 & 0.019 & -0.027 to 0.065 \\
\hline 14 & 0.055 & -0.011 to 0.121 & 0.026 & -0.021 to 0.073 & 0.027 & -0.017 to 0.070 \\
\hline 15 & 0.076 & 0.000 to 0.152 & 0.057 & 0.011 to 0.103 & 0.017 & -0.034 to 0.069 \\
\hline 16 & 0.028 & -0.049 to 0.104 & 0.025 & -0.031 to 0.081 & 0.002 & -0.041 to 0.044 \\
\hline 17 & 0.065 & 0.005 to 0.126 & 0.036 & -0.020 to 0.092 & 0.026 & -0.005 to 0.057 \\
\hline 18 & 0.070 & -0.024 to 0.165 & 0.038 & -0.041 to 0.118 & 0.029 & -0.007 to 0.065 \\
\hline \multicolumn{7}{|c|}{ Participants on long-term benefits } \\
\hline 12 & 0.074 & 0.011 to 0.137 & 0.002 & -0.042 to 0.047 & 0.058 & 0.002 to 0.115 \\
\hline 13 & 0.096 & 0.025 to 0.168 & 0.025 & -0.025 to 0.075 & 0.058 & -0.003 to 0.119 \\
\hline 14 & 0.087 & -0.004 to 0.178 & 0.029 & -0.044 to 0.103 & 0.048 & -0.016 to 0.112 \\
\hline 15 & 0.111 & 0.019 to 0.204 & 0.044 & -0.041 to 0.129 & 0.056 & -0.013 to 0.124 \\
\hline 16 & 0.120 & 0.009 to 0.230 & 0.058 & -0.043 to 0.159 & 0.054 & -0.009 to 0.116 \\
\hline 17 & 0.159 & 0.060 to 0.258 & 0.081 & 0.012 to 0.151 & 0.065 & -0.010 to 0.141 \\
\hline 18 & 0.178 & 0.104 to 0.253 & 0.091 & 0.033 to 0.149 & 0.066 & 0.004 to 0.127 \\
\hline
\end{tabular}

Separate regression analyses for each calendar month after inclusion. Controls for gender, age, marital status, income prior to inclusion, self-assessed health, expectation of return to work, work status at inclusion and treatment site. $95 \%$ Cls based on robust SEs allowing for correlated residuals within treatment sites. 
Table 3 Mean (SE) scores of secondary outcomes after 12 months follow-up*

\begin{tabular}{|c|c|c|c|c|c|c|c|}
\hline Outcome & Group & $\mathbf{N}$ & Mean & SE & $95 \% \mathrm{Cl}$ & t Test & pValue \\
\hline \multirow[t]{2}{*}{ HAD total } & $\mathrm{AWaC}$ & 376 & 13.00 & 0.43 & 12.14 to 13.84 & $t=3.14, d f=625$ & 0.002 \\
\hline & Control & 251 & 15.12 & 0.53 & 14.08 to 16.16 & & \\
\hline \multirow[t]{2}{*}{ Depression (HAD-D) } & $\mathrm{AWaC}$ & 376 & 5.11 & 0.23 & 4.67 to 5.56 & $\mathrm{t}=3.23, \mathrm{df}=625$ & 0.001 \\
\hline & Control & 251 & 6.27 & 0.28 & 5.72 to 6.81 & & \\
\hline \multirow[t]{2}{*}{ Anxiety (HAD-A) } & $\mathrm{AWaC}$ & 376 & 7.88 & 0.24 & 7.40 to 8.36 & $\mathrm{t}=2.52, \mathrm{df}=625$ & 0.012 \\
\hline & Control & 251 & 8.86 & 0.30 & 8.26 to 9.46 & & \\
\hline \multirow[t]{2}{*}{ Health-related quality of life (EQ5D) } & $\mathrm{AWaC}$ & 376 & 65.64 & 1.15 & 63.38 to 67.90 & $\mathrm{t}=-2.24, \mathrm{df}=616$ & 0.026 \\
\hline & Control & 251 & 61.57 & 1.41 & 58.78 to 64.36 & & \\
\hline
\end{tabular}

The probability of receiving IPS was estimated by logistic regression including employment status at baseline and other individual characteristics and site as regressors. Persons on longterm benefits had a higher probability of receiving IPS than the other subgroups. The probability of receiving IPS was also higher if scoring $\geq 8$ on HADS anxiety at inclusion. The centres varied in terms of job support service delivery.

\section{Cost-benefit analyses}

Total costs at the six centres included operating expenses, wage payments and payment for job support services. In 2011, this amounted in total to NoK 17246 million (about $£ 1883040$ ). Divided by the expected number of treated patients per year under normal operating conditions (615), this equates to NoK 28043 (about $£ 3062$ ) per patient for AWaC. In addition, costs for society through other services provided by NAV and/or healthcare providers amounted to NoK 9469 (about £1034) for each person receiving AWaC (see online supplementary table S2). Persons referred to treatment as usual received more services from NAV and had more consultations with psychologists and other healthcare providers than participants in the treatment group. In total, costs of treatment per person receiving treatment as usual amounted to NoK 16800 (about $£ 1834$ ). Taking the difference and adjusting for marginal costs of public funds (1 $+0.2) \times(37512-16800)$, additional costs for the society from participation in AWaC amounted to NoK 24854 per person (about $£ 2713$ ).

Benefits emerged from observed between-group differences in full RTW (ie, not receiving any sickness benefits) (0.0456) and partly $(0.025)$ RTW. The net social value of production per person per year, based on average annual earnings of participants in the sample (NoK 340400$)$ and adding social costs, was $(399664-76496+0.2 \times 350240) \quad$ NoK 393216 when there was full work participation, and multiplied by 0.5 in the case of partly return to work. Based on this, the average per person increase in the net present value of production from participation in AWaC amounted to $((0.044 \times 393216) / 1.04+0 \times 5$ $(0.024 \times 393$ 216)/1.04) NoK 21173 (about £2311). Under the assumption that treatment effects would last for 1 year, economic benefits did not outweight additional costs of participation in the AWaC programme. On the basis of overall treatment effects of the assumed 615 persons in treatment/year, the estimated net present value of productivity gains was negative NoK 2263815 (about $£ 247141$ ).

Had AWaC been given only to persons on long-term benefits, costs for additional services by NAV would have been considerably higher in both treatment groups. The stronger treatment effects, both in terms of full-time work (0.077) and part time work $(0.072)$, would, however, generate productivity gains to more than outweight the additional costs of treatment. In total, the net present value of the productivity gains with treatment effects lasting for 1 year amounted to NoK 4731810 (about $£ 516570)$.

\section{DISCUSSION}

The main aim of this project was to evaluate if the AWaC programme, a systematic approach integrating elements from CBT and IPS, led to increased or maintained work participation for people with common mental disorders. The results indicated a significant effect in favour of $\mathrm{AWaC}$ at 12 -month follow-up. In the group that received $\mathrm{AWaC}, 44 \%$ were fully or partially at work at 12 months, compared with $37 \%$ in the control group. The main effect remained at 18 months. Further, the effect was stronger among those on long-term benefits, with $24 \%$ increased or maintained work participation in the AWaC group compared with $12 \%$ in the control group at 12 months, a difference that increased to $30 \%$ versus $11 \%$ at 18 months. The AWaC group also had a more favourable development in terms of mental health status and health-related quality of life after 12 months. We observed no corresponding larger effect difference for the group on long-term benefits in terms of symptoms of mental health or health-related quality of life.

The stronger effect difference for the subgroup on long-term benefits might have to do with both the intervention better matching the actual needs for this group, but can also reflect less optimal services in the control condition for this group. Whether the stronger results were caused by specific elements in the intervention cannot be answered by the trial, but we did find a higher probablity of receiving job support in the subgroup on long-term benefits. This indicates that work is correlated to job support, but it is impossible to distinguish between a potential causal effect and correlation coming from job support being given based on individual characteristics that in and of itself could be correlated to work.

The cost-benefit analyses did not demonstrate positive economic returns of the AWaC programme. However, for the subgroup on long-term benefits, the stronger effect sizes translated to a large positive economic net return. These cost-benefit estimates were all based on the assumption of a treatment effect lasting for 1 year. To the extent a treatment effect outlasts this 1 year assumption, the programme will yield a higher economic return. Furthermore, we acknowledge that increased employment not only brings production gains, but also can promote health and quality of life for the individual. While the latter effects are not included in the strict economic analysis, these important gains would certainly add to the monetary figures. 
Earlier this year, the Organization for Economic Cooperation and Development (OECD) stated that Norway has the highest level of sickness absence and costs related to lost labour among all the member countries. ${ }^{1}$ Common mental disorders contribute most to the recent rise in expenditures. The report further concludes that the Norwegian system can contribute to exclusion of people with mental disorders through 'welfare-traps' of disincentives. This context is important to bear in mind when interpreting the results. The Norwegian welfare system, with $100 \%$ compensation of income from day one and a high level of available services at low cost for the individual, stands out from other countries. As such, the system can influence the achievable effect sizes for interventions in this area. Few previous intervention projects aiming to increase RTW have been able to demonstrate significant results, ${ }^{28-30}$ which makes the present effect stand out as profound and particularly clinically important in the Norwegian context.

\section{Strengths and limitations}

The main strength of the study is the multicentre RCT design and the large sample size, providing robust data on the effect of the intervention as deployed in an ecologically valid setting. We included participants who were struggling, but still working, and people who were out of work and further away from employment, as both extremes would be potential targets for the model if further implemented. The research group was not involved in running the sites, or in patient treatment, which ensured an independent evaluation. A key strength was that loss to follow-up for the main outcomes was eliminated through the use of registry data.

National registry data provided information about all social security benefits received during the project period, work participation and vocational rehabilitation services. There was, however, no registry information available for health outcomes such as use of health services or health status. Data on services outside those directly related to work participation therefore relied on self-report, with corresponding potential for measurement error and non-response bias. Staff adherence towards the IPS components was not evaluated during the project, as development and improvement towards the gold standard of IPS for this new target group was ongoing throughout the project period. Adherence to the eight IPS principles was, however, continuously pursued, and any diversions from the IPS principles are explained in the attached study protocol (see online supplementary file).

We chose not to measure staff adherence to the CBT treatment protocol, as the aim was to test the intervention in a natural setting, rather than to adhere to a stricter efficacy trial. Streamlining of the intervention was promoted through monthly seminars for the teams at the centres, with courses delivered from approved supervisors within the Norwegian Association for Cognitive Therapy.

Previous research shows that CBT reduces symptoms and improves function for patients with common mental disorders. ${ }^{31-34}$ There is some previous evidence that CBT focusing on work has an effect on reduced sickness absence, ${ }^{35}$ while studies with a less pronounced focus on work resumption are lacking RTW effects. A few previous studies of CBT interventions have indeed failed to demonstrate an increased RTW effect in common mental disorders. ${ }^{36-39}$ A key difference between those studies and this study is, however, the explicit focus on work, and the integration of work-focused CBT and individual job support. No previous studies have ever combined and integrated these two elements for people with common mental disorders. The current trial is the first large-scale randomised controlled study to demonstrate an effect of a behavioural intervention on work participation for the large group of workers with common mental disorders.

\section{Implications for research and practice}

A significant implication for both clinicians and those who struggle with work participation due to common mental disorders is the demonstration of an adequate, efficient and available intervention. Such interventions have previously been lacking or at least not delivered systematically. ${ }^{40}$ Through the AWaC programme, participants not only receive support resulting in increased work participation, but also experience improvements in mental health and health-related quality of life. In line with recent OECD recommendations, ${ }^{1}$ the $\mathrm{AWaC}$ programme integrates mental healthcare and rehabilitation services, and incorporates concerns for both work and health.

The AWaC trial was the first RCT commisioned by the Norwegian health and welfare authorities. This initiative is unique in a Norwegian (and probably also international) context. The experience of successful collaboration between academic researchers and policymakers, where a novel policy scheme and intervention underwent rigorous scientific evaluations before large-scale implementation, has important policy implications and should serve as an inspiration for further use of trials in this sector. Beyond strengthening the evidence base, such collaborations can help bridge the gap between knowledge and practical application. ${ }^{41}$

Our main recommendation for future research is thus to run more trials of this kind to rapidly improve our evidence base on this vital topic. We recommend using registry-based outcomes where available, with long-term follow-up whenever possible, and also further trials and replications where combinations of CBT and job support are offered. Our results, and the rapidly growing IPS literature for severe mental disorders, suggest that this might be a viable route to reduced workforce dropout from mental disorders.

Common mental disorders are not just an individual clinical problem; they are also a major challenge for stakeholders in both the work and health sectors and the society at large. A combined model of work-focused CBT and individual job support was more effective than usual care in increasing or maintaining work participation for people with common mental disorders. The effects were profound for people on long-term benefits, and translated to a major net value of productivity gains for this group.

Correction notice This paper has been amended since it was published Online First. Camilla Lovvik's affiliations have been corrected from 1,2 to 1,4.

Twitter Follow Silje Reme at @siljeendresen

Contributors SER and CL were responsible for the general coordination of the study. ALG, SAL and SØ were responsible for the data analyses. All the authors designed the study, helped write the manuscript, and read and approved the final version of the manuscript. SER and ALG had full access to all the data in the study and take responsibility for the integrity of the data and the accuracy of the data analysis

Funding The project was commissioned by the Norwegian Ministry of Health and Ministry of Labour, and financed through the National Strategy on Work and Mental Health (2007-2012)

Competing interests None declared.

Patient consent Obtained.

Ethics approval The Norwegian Regional Ethical Committee approved the study (reference number: 2010/1130).

Provenance and peer review Not commissioned; externally peer reviewed.

Open Access This is an Open Access article distributed in accordance with the Creative Commons Attribution Non Commercial (CC BY-NC 4.0) license, which 
permits others to distribute, remix, adapt, build upon this work non-commercially, and license their derivative works on different terms, provided the original work is properly cited and the use is non-commercial. See: http://creativecommons.org/ licenses/by-nc/4.0/

\section{REFERENCES}

1 OECD. Mental health and work. OECD Publishing, Norway, 2013.

2 Cattrell A, Harris EC, Palmer KT, et al. Regional trends in awards of incapacity benefit by cause. Occup Med (Lond) 2011;61:148-51.

3 Wittchen HU, Jacobi F, Rehm J, et al. The size and burden of mental disorders and other disorders of the brain in Europe 2010. Eur Neuropsychopharmacol 2011;21:655-79.

4 Kessler RC, Demler 0, Frank RG, et al. Prevalence and treatment of mental disorders, 1990 to 2003. N Engl J Med 2005;352:2515-23.

5 Spiers N, Bebbington P, McManus S, et al. Age and birth cohort differences in the prevalence of common mental disorder in England: National Psychiatric Morbidity Surveys 1993-2007. Br J Psychiatry 2011;198:479-84.

6 Office for National Statistics. Psychiatric morbidity among adults living in private households. London: The Stationery Office, 2001.

7 Henderson M, Harvey SB, Overland S, et al. Work and common psychiatric disorders. J R Soc Med 2011;104:198-207.

8 Waddell G, Burton K, Aylward M. Work and common health problems. J Insur Med 2007:39:109-20.

9 Blank L, Peters J, Pickvance S, et al. A systematic review of the factors which predict return to work for people suffering episodes of poor mental health. J Occup Rehabil 2008;18:27-34.

10 National Institute for Health and Care Excellence. Depression in adults: NICE guidance. NICE, 2009.

11 National Institute for Health and Care Excellence. Anxiety: NICE guidance. NICE, 2011.

12 Campbell K, Bond GR, Drake RE. Who benefits from supported employment: a meta-analytic study. Schizophr Bull 2011;37:370-80.

13 Burns T, Catty J, White $S$, et al. The impact of supported employment and working on clinical and social functioning: results of an international study of individual placement and support. Schizophr Bull 2009;35:949-58.

14 Bond GR. Supported employment: evidence for an evidence-based practice. Psychiatr Rehabil J 2004;27:345-59.

15 Bond GR, Drake RE, Becker DR. An update on randomized controlled trials of evidence-based supported employment. Psychiatr Rehabil J 2008;31:280-90.

16 Becker D, Whitley R, Bailey EL, et al. Long-term employment trajectories among participants with severe mental illness in supported employment. Psychiatr Serv 2007:58:922-8.

17 Rai D, Skapinakis $\mathrm{P}$, Wiles $\mathrm{N}$, et al. Common mental disorders, subthreshold symptoms and disability: longitudinal study. Br J Psychiatry 2010;197:411-12.

18 Bond GR, Drake RE. Making the case for IPS supported employment. Adm Policy Ment Health 2014;41:69-73.

19 Zigmond AS, Snaith RP. The hospital anxiety and depression scale. Acta Psychiatr Scand 1983;67:361-70.

20 Bjelland I, Dahl AA, Haug TT, et al. The validity of the Hospital Anxiety and Depression Scale. An updated literature review. J Psychosom Res 2002;52:69-77.

21 The EuroQol Group. EuroQol-a new facility for the measurement of health-related quality of life. Health Policy 1990;16:199-208.

22 Seaman SR, White IR. Review of inverse probability weighting for dealing with missing data. Stat Methods Med Res 2013;22:278-95.
23 Barber JP, Liese BS, Abrams MJ. Development of the cognitive therapy adherence and competence scale. Psychother Res 2003;13:205-221.

24 Bond GR, Peterson AE, Becker DR, et al. Validation of the Revised Individual Placement and Support Fidelity Scale (IPS-25). Psychiatr Serv 2012;63:758-63.

25 Haveman RH, Halberstadt V, Burhauser RV. Public policy toward disabled workers. Cross-national analyses of economic impacts. Ithaca: Cornell University Press, 1984.

26 Berkowitz M. Measuring the effects of public programs. Cost and benefits in vocational rehabilitation. Philadelphia: Temple University Press, 1988.

27 Risa AE. Economic evaluation of vocational rehabilitation programs. In: de Jong PR, Marmor TR, eds. Social policy and the labor market. Aldershot, Aldershot, 1997:191-203.

28 Eriksen HR, Ihlebaek C, Mikkelsen A, et al. Improving subjective health at the worksite: a randomized controlled trial of stress management training physical exercise and an integrated health programme. Occup Med (Lond) 2002:52:383-91.

29 Magnussen L, Strand LI, Skouen JS, et al. Motivating disability pensioners with back pain to return to work - a randomized controlled trial. J Rehabil Med 2007;39:81-7.

30 Tveito TH, Eriksen HR. Integrated health programme: a workplace randomized controlled trial. J Adv Nurs 2009;65:110-19.

31 Hofmann SG, Smits JA. Cognitive-behavioral therapy for adult anxiety disorders: a meta-analysis of randomized placebo-controlled trials. J Clin Psychiatry 2008;69:621-32

32 Spek V, Cuijpers $P$, Nyklicek I, et al. Internet-based cognitive behaviour therapy for symptoms of depression and anxiety: a meta-analysis. Psychol Med 2007:37:319-28

33 Wiles N, Thomas L, Abel A, et al. Cognitive behavioural therapy as an adjunct to pharmacotherapy for primary care based patients with treatment resistant depression: results of the CoBalT randomised controlled trial. Lancet 2013;381:375-84.

34 Hollon SD, DeRubeis RJ, Shelton RC, et al. Prevention of relapse following cognitive therapy vs medications in moderate to severe depression. Arch Gen Psychiatry 2005;62:417-22.

35 Lagerveld SE, Blonk RW, Brenninkmeijer V, et al. Work-focused treatment of common mental disorders and return to work: a comparative outcome study. J Occup Health Psychol 2012;17:220-34.

36 de Vente W, Kamphuis JH, Emmelkamp PM, et al. Individual and group cognitive-behavioral treatment for work-related stress complaints and sickness absence: a randomized controlled trial. J Occup Health Psychol 2008;13:214-31

37 Blonk RWB, Brenninkmeijer V, Lagerveld SE, et al. Return to work: a comparison of two cognitive behavioural interventions in cases of work-related psychological complaints among the self-employed. Work Stress 2006;20:129-44.

38 Willert MV, Thulstrup AM, Bonde JP. Effects of a stress management intervention on absenteeism and return to work - results from a randomized wait-list controlled trial. Scand J Work Environ Health 2011;37:186-95.

39 Stenlund T, Ahlgren C, Lindahl B, et al. Cognitively oriented behavioral rehabilitation in combination with Qigong for patients on long-term sick leave because of burnout: REST—a randomized clinical trial. Int J Behav Med 2009;16:294-303.

40 Overland S, Glozier N, Krokstad S, et al. Undertreatment before the award of a disability pension for mental illness: the HUNT study. Psychiatr Serv 2007:58:1479-82.

41 Smith PM. A transdisciplinary approach to research on work and health: what is it, what could it contribute, and what are the challenges? Crit Public Health 2007:17:159-69. 